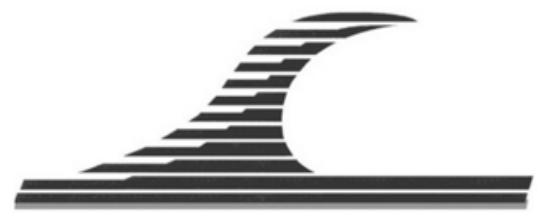

Revue Paralia, Volume 6 (2013) pp 11.1-11.14

Mots-clés : Sédiment, Granulométrie, Dynamique sédimentaire, Lagune de Bizerte, Tunisie.

(C) Editions Paralia CFL

\title{
Etude de l'hydrodynamique sédimentaire de la lagune de Bizerte
}

\section{Mouldi BRAHIM ${ }^{1}$, Abdelfattah ATOUI ${ }^{1}$, Béchir BEJAOUI ${ }^{1}$}

1. Institut National des Sciences et Technologies de la Mer, 28 rue 2 mars 1934, Salammbô, 2025 Tunis, Tunisie.

mouldi.brahim@instm.rnrt.tn

\section{Résumé :}

Dans le cadre du projet de recherche MODEM intitulé "Modélisation Opérationnelle pour le Développement des Écosystèmes Marins", un travail de recherche a été effectué dans la lagune de Bizerte portant sur l'hydrodynamique sédimentaire. Les analyses granulométriques des sédiments de surface, prélevés à différentes profondeurs, ont permis d'identifier la nature de ces sédiments et de préciser les facteurs et les phénomènes qui interviennent dans leur transport et leur dépôt. Elles ont montré que les sédiments provenant de l'érosion des côtes sont soumis à une dynamique qui est contrôlée essentiellement par l'action des courants étroitement liés aux vents. Ces courants sont assez importants dans les petites profondeurs au nord et au sud, mais ils sont faibles dans les grandes profondeurs à l’intérieur de la lagune.

Soumis le 23 novembre 2010, accepté le 20 septembre 2013, en ligne le 23 décembre 2013.

La seule version examinée est celle écrite en français. La ou les autres versions n'étant pas examinées par le comité de rédaction de la revue, sont donc publiées sous l'entière responsabilité du ou des auteurs.

A TRANSLATED VERSION IN ENGLISH IS AVAILABLE ONLINE

Pour citer cet article :

BRAHIM M., ATOUI A., BEJAOUI B. (2013). Étude de l'hydrodynamique sédimentaire de la lagune de Bizerte. Revue Paralia, Vol. 6, pp 11.1-11.14.

DOI:10.5150/revue-paralia.2013.011 ～(disponible en ligne - http://www.paralia.fr - available online) 


\section{Introduction}

La lagune de Bizerte est située à l'extrême nord de la Tunisie, entre $37^{\circ} 08^{\prime}$ et $37^{\circ} 15^{\prime}$ de latitude Nord et entre $9^{\circ} 45^{\prime}$ et $9^{\circ} 57^{\prime}$ de longitude Est (figure 1). C'est une dépression de $128 \mathrm{~km}^{2}$ connue par sa position géostratégique, car elle est reliée, d'une part à la mer Méditerranée par un chenal de navigation d'environ $1500 \mathrm{~m}$ de long, $300 \mathrm{~m}$ de large et $12 \mathrm{~m}$ de profondeur et d'autre part au lac Ichkeul par un chenal étroit et sinueux appelé Oued Tinja d'environ 5 km de long (ANPE, 1990 ; OUAKAD, 1982 et 1993).

La lagune reçoit plusieurs cours d'eau (figure 1). Elle est limitée au nord par de petits bassins côtiers, au sud par la basse vallée de la Medjerda, à l'est par les bassins versants de la région côtière de Ras El Djebel et à l'ouest, par la Garaât Ichkeul avec laquelle elle communique par l'oued Tinja qui alimente la lagune de Bizerte en eau pendant la saison pluvieuse (KALLEL, 1989). L'équilibre naturel entre les apports d'eau douce et les échanges avec la mer a permis à la lagune de Bizerte de connaître une riche biodiversité. Depuis le début du siècle dernier, cette lagune est utilisée pour l'aquaculture et la pêche. Les premiers essais de conchyliculture ont été réalisés en 1952 dans la zone nord-est de la lagune et ont concerné l'espèce Crassostrea angulata. A partir de 1963, cette activité a été relayée par la mise en culture des moules (Mytilus galloprovincialis). Selon GIMAZANE (1981); la croissance de ces moules a été très bonne et elle est comparable à celle des moules de l'étang de Thau (France). L'élevage des moules a été bien développé à partir des années 80 dans la lagune (GREDOPAR, 1986). Cependant depuis plusieurs années on assiste à un développement urbain sur les bords de la lagune, caractérisé par l'implantation de sites industriels importants comme le complexe sidérurgique "El Fouledh", les constructions navales de la SOCOMENA à Menzel Bourguiba et la cimenterie de Bizerte. Des aménagements ont touché les bassins versants : installation de plusieurs barrages au niveau des oueds à l'amont du lac Ichkeul et du bassin versant de la lagune. Parmi ces barrages nous pouvons citer le barrage de Sejenane construit en 1994, le barrage de Ghezala construit en 1984, et les deux barrages de Melah et de Douimis installés récemment en 2005 (DGBGTH, 2006). Ces aménagements ont affecté l'équilibre naturel faisant varier le bilan hydrique. Le total des apports d'eau douce a ainsi chuté de $270 \times 10^{6} \mathrm{~m}^{3} \mathrm{an}^{-1}$ à seulement $120 \times 10^{6} \mathrm{~m}^{3}$ $\mathrm{an}^{-1}$. Le bilan hydrique est donc devenu tributaire des programmes de gestion de l'ensemble de l'infrastructure mise en place. Ces aménagements ont engendré des modifications profondes des caractéristiques hydrologiques de la lagune et en particulier de la salinité (HARZALLAH, 2003).

Les principales sources de sédiments dans la lagune de Bizerte, sont d'après KAMENS et al. $(1984$; 1989) : (1) les boues non calcaires et les sables charriés par l’oued Tinja en provenance du lac Ichkeul, (2) les sables issus des organismes marins, (3) les calcaires et les matériaux solides résultant de l'érosion du sol des terres cultivées environnantes et amenés par les cours d'eau (figure 1), (4) enfin et de moindre importance, les apports 
éoliens (silts et composés argileux) et les apports en provenance de la Méditerranée (KAMENS et al., 1984).

Le climat de la région de Bizerte est de type méditerranéen avec un été chaud et sec et un hiver doux et pluvieux.

La région de Bizerte est caractérisée par des vents qui soufflent généralement du secteur ouest, et particulièrement ceux du nord-ouest, sont les plus fréquents. Ils soufflent à des vitesses comprises entre 5 et $9 \mathrm{~m} \mathrm{~s}^{-1}$ pouvant atteindre $15 \mathrm{~m} \mathrm{~s}^{-1}$. Les vents d'est sont moins fréquents. Les vents qui soufflent du secteur sud-est en période estivale appelés Sirocco sont généralement secs et chauds (HIDROTECNICA PORTUGUESA, 1995).

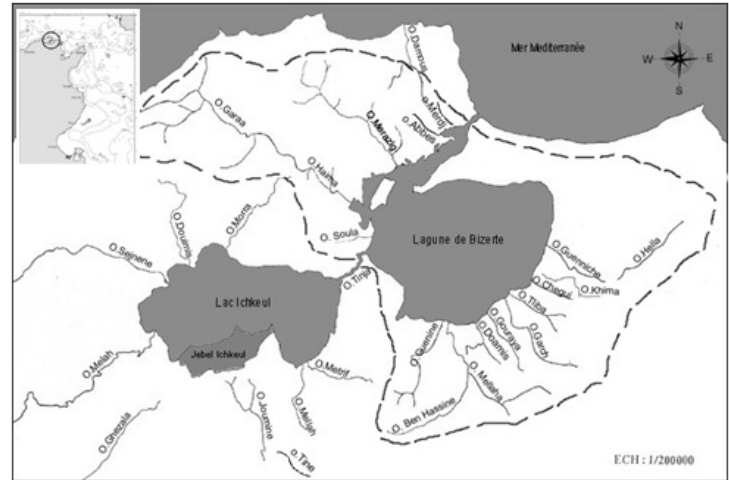

Figure 1. Situation géographique de la lagune de Bizerte et limite (---) du bassin versant.

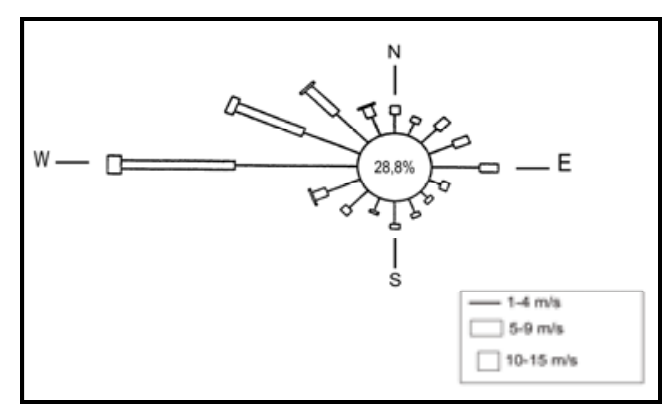

Figure 2. Rose des vents soufflant dans la région de Bizerte (HIDROTECNICA PORTUGUESA, 1995).

Les données hydrodynamiques (HARZALLAH, 2003 ; BEJAOUI \& HARZALLAH, 2005 ; BEJAOUI, 2009) ont permis d'avancer que le secteur d'étude est une zone de haute énergie. La houle favorise non seulement le brassage des sédiments mais aussi la mobilisation permanente des dépôts superficiels.

Les travaux de SOUSSI (1981) et KAMENS et al. (1984) montrent que la répartition des sédiments dans la lagune de Bizerte est fonction des sources d'apport et de la morphologie du fond du lac (voir carte 1 ). On distingue les faciès suivants :

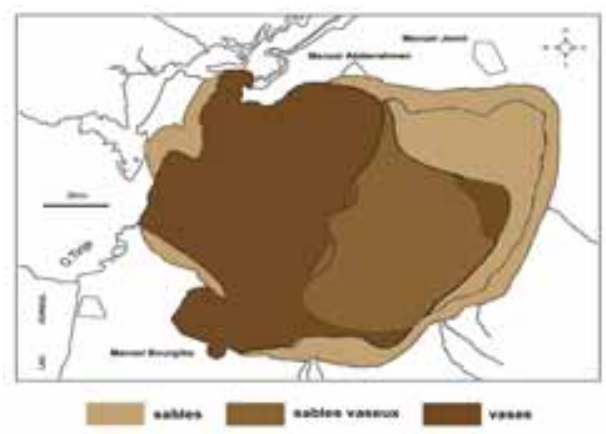

Carte 1. Répartition des sédiments au niveau de la lagune de Bizerte (SOUSSI, 1981). 
- Faciès sableux dont plus de 50\% du dépôt sont formés par la fraction grossière $(>63 \mu \mathrm{m})$.

- Faciès silteux à argilo-silteux caractérisant la partie orientale de la lagune et dont 20 à $50 \%$ du sédiment sont représentés par la fraction comprise entre 2 et $63 \mu \mathrm{m}$.

- Faciès argileux : occupant le centre du bassin ainsi que sa partie ouest, où la fraction inférieure à $2 \mu \mathrm{m}$ forme plus de 50\% du dépôt.

Ainsi dans le cadre du projet MODEM, un travail de recherche a été effectué, lequel a pour objectif : l'estimation des caractéristiques hydrodynamiques de la lagune de Bizerte à partir d'analyses granulométriques, la comparaison avec des données antérieures et avec une simulation numérique des courants.

\section{La modélisation de la circulation des eaux}

Les seuls travaux sur la circulation des eaux sont ceux effectués par HARZALLAH (2003), BEJAOUI et HARZALLAH (2005), BEJAOUI et al. (2008) et BEJAOUI (2009), par la modélisation hydrodynamique de la circulation des eaux.

Le modèle hydrodynamique appliqué dans la lagune de Bizerte est basé sur les équations primitives de la circulation et de la thermodynamique écrites en coordonnées sigma (BLUMBERG \& MELLOR, 1987). La grille du modèle est curviligne orthogonale de type Arakawa C. Elle est constituée de 61×52 mailles et de 11 niveaux verticaux. L'espacement suivant la longitude varie entre 75 et $495 \mathrm{~m}$ et celui suivant la latitude est compris entre 218 et 686 m (BEJAOUI et al., 2008).

Les eaux de surface, soumises à des tensions du vent du secteur nord-ouest (moyenne des mois de décembre, janvier et février), se déplacent vers le sud-est pour toutes les régions de la lagune. Les courants les plus forts se trouvent au niveau des bords nord, ouest et sud. Au centre de la lagune, les courants sont relativement faibles. Au fond, l'effet du vent diminue et le sens de la circulation s'inverse vers la direction nord-ouest. L'eau ramenée vers la direction sud-est, par cisaillement du vent, retourne vers le nordouest en traversant le centre de la lagune (HARZALLAH, 2003 ; BEJAOUI, 2009).

La simulation en été (moyenne des mois de juin, juillet et août) des courants de surface, des courants de fond et de la composante barotrope du courant est similaire à celle de la circulation hivernale. Néanmoins, l'intensité des courants faiblit en conséquence de la diminution de celle du vent. Notons que le vent moyen pendant la période estivale est aussi du nord-ouest ce qui justifie la similarité de la structure des courants pendant les deux périodes estivale et hivernale (BEJAOUI, 2009).

Une particularité importante de la circulation des eaux est l'apparition de trois cellules de circulation giratoire. Une située au nord, une située au sud et une troisième moins importante située à l'ouest (figure 5). L’échelle au niveau des figures correspond à l'intensité et au sens de l'écoulement. Les valeurs positives indiquent que le sens de l'écoulement est contraire au sens des aiguilles d’une montre. Les valeurs négatives indiquent le sens opposé. 


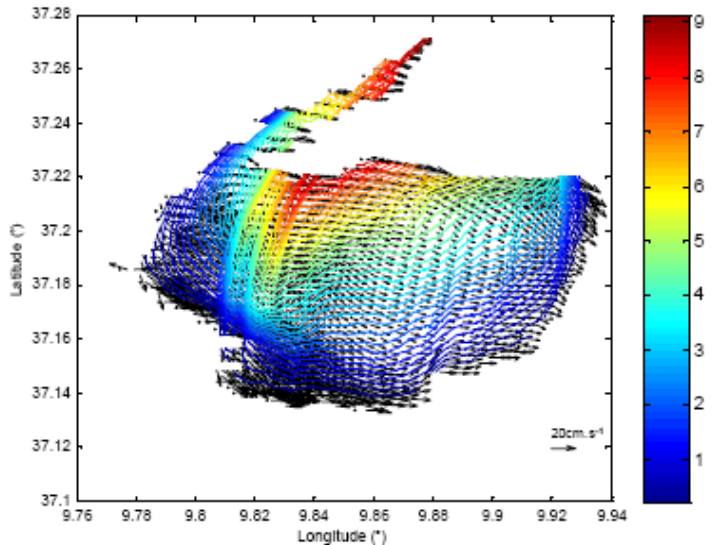

Figure 3. Circulation moyenne hivernale en surface générée par un vent du secteur nord-ouest (BEJAOUI, 2009)

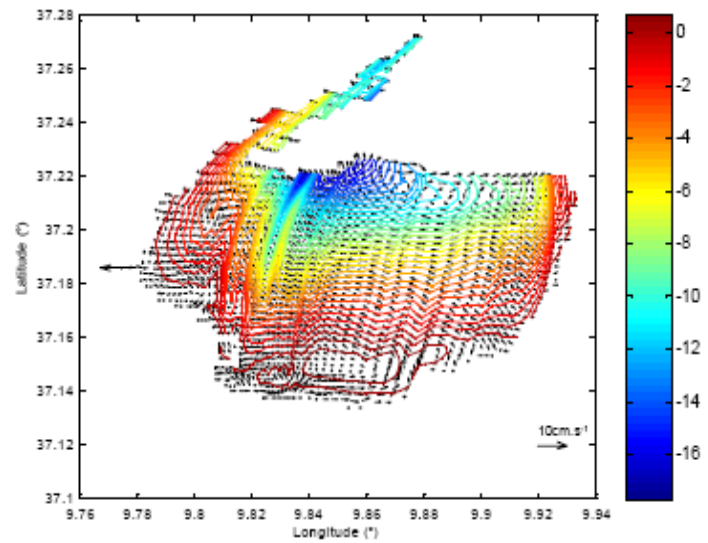

Figure 4. Circulation moyenne hivernale près du fond générée par un vent $d u$ secteur nord-ouest (BEJAOUI, 2009)

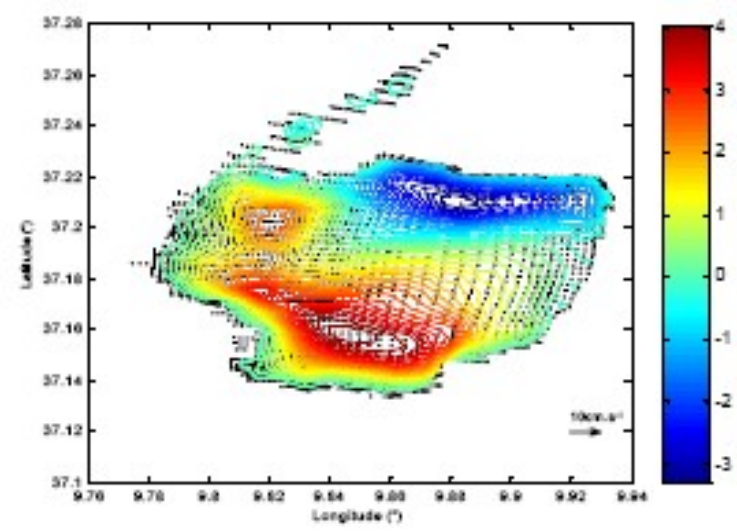

Figure 5. Circulation moyenne hivernale barotrope ainsi que les lignes de courant (en Sv ou $\left.10^{6} \mathrm{~m}^{3} \mathrm{~s}^{1}\right)$. Mise en évidence de trois cellules giratoires au nord, au sud et à l'ouest. (BEJAOUI, 2009).

\section{Matériels et méthodes}

Deux campagnes d'échantillonnage des sédiments de surface de la lagune de Bizerte ont été effectuées durant le mois de septembre 2007. La première a été effectuée les 6 et 7 septembre et la seconde les 12 et 13 septembre. Au total 44 échantillons de sédiment de surface ont été prélevés à des profondeurs d'eau variables couvrant l'ensemble de la lagune (figure 6). L'échantillonnage a été réalisé à l'aide d'une benne de type Van Veen et la localisation des positions des points de prélèvement a été effectuée à l'aide d'un GPS radar. Les sédiments fins vaseux ont été tamisés par voie humide sur un tamis de maille $63 \mu \mathrm{m}$. Ce tamisage humide a permis de séparer la fraction grossière (diamètre $>63 \mu \mathrm{m}$ ) de la fraction fine (diamètre<63 $\mu \mathrm{m}$ ). Les échantillons des sédiments dont les tailles sont supérieures à $63 \mu \mathrm{m}$, ont été séchés à $50{ }^{\circ} \mathrm{C}$. Ils ont été ensuite tamisés par voie sèche durant 20 minutes sur une série de tamis de type AFNOR dont 
les mailles varient de $2000 \mu \mathrm{m}$ à $63 \mu \mathrm{m}(2000 \mu \mathrm{m}, 1400 \mu \mathrm{m}, 630 \mu \mathrm{m}, 500 \mu \mathrm{m}, 250 \mu \mathrm{m}$, $180 \mu \mathrm{m}, 125 \mu \mathrm{m}, 100 \mu \mathrm{m}$ et $63 \mu \mathrm{m})$.

L'analyse de la micro granulométrie a été réalisée par un granulomètre LASER de type Mastersizer 2000. L'échantillon est aspiré par une pompe agitatrice vers le Mastersizer 2000. Les résultats sont fournis sous une forme graphique et numérique de la taille des grains en $\mu \mathrm{m}$ et du pourcentage cumulé en volume.

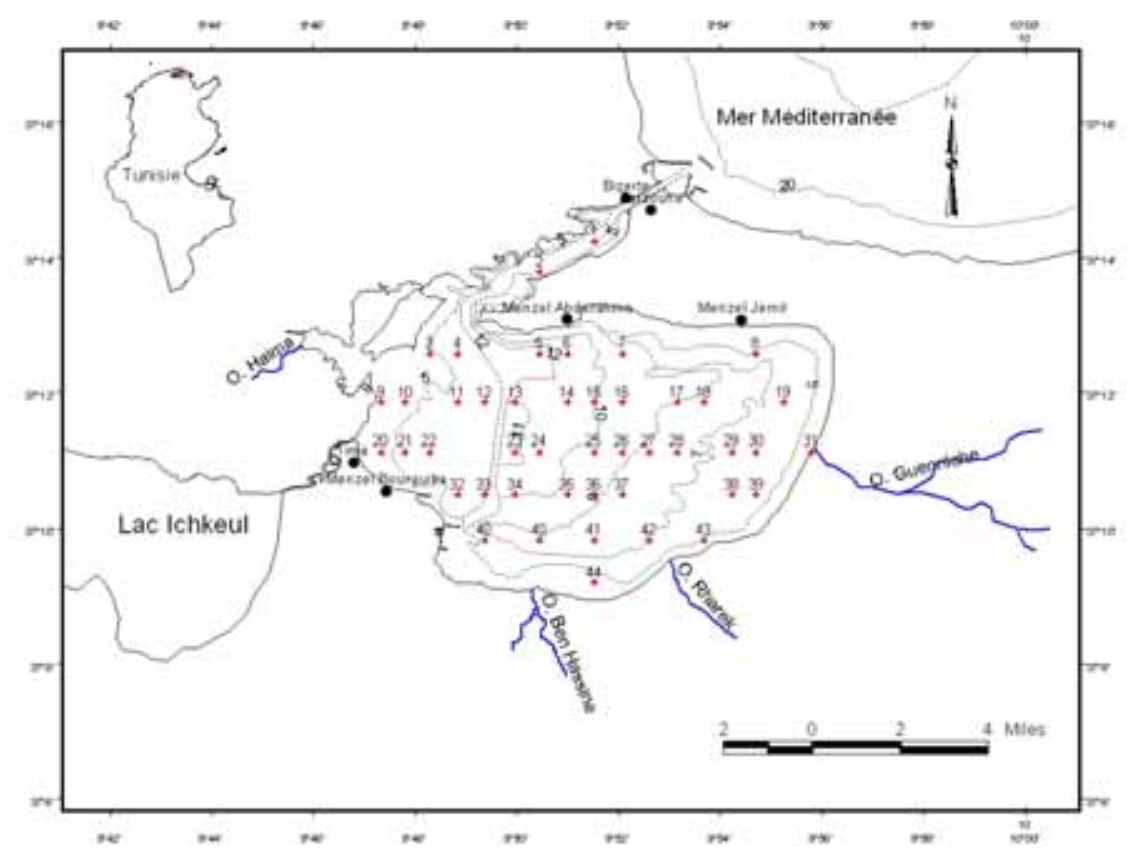

Figure 6. Localisation des stations de prélèvement de sédiments et bathymétrie de la lagune de Bizerte.

\section{Résultats des analyses granulométriques}

\subsection{Répartition des sédiments de surface}

Nous avons calculé le pourcentage de la fraction grossière $(>63 \mu \mathrm{m})$ et de la fraction fine $(<63 \mu \mathrm{m})$. Les résultats obtenus montrent une importante variation du taux des deux fractions : en effet, le pourcentage de la fraction fine varie entre 4.6 et $100 \%$.

Dans la lagune de Bizerte, la répartition de la fraction fine et de la fraction grossière a permis de distinguer des sédiments formés essentiellement de sables, de sables vaseux, de vase sableuse ou de vase. La granulométrie des sédiments de surface est répartie suivant quatre zones distinctes (figure 7) :

- (1) Les secteurs profonds de la lagune sont caractérisés par des sédiments vaseux fins comme également le secteur ouest et plus particulièrement celui soumis à l'influence directe des apports solides des oueds drainant les bassins versants qui entourent la dépression formée par le lac Tinja au sud jusqu’à la station conchylicole de Menzel Jamil à l'est. 


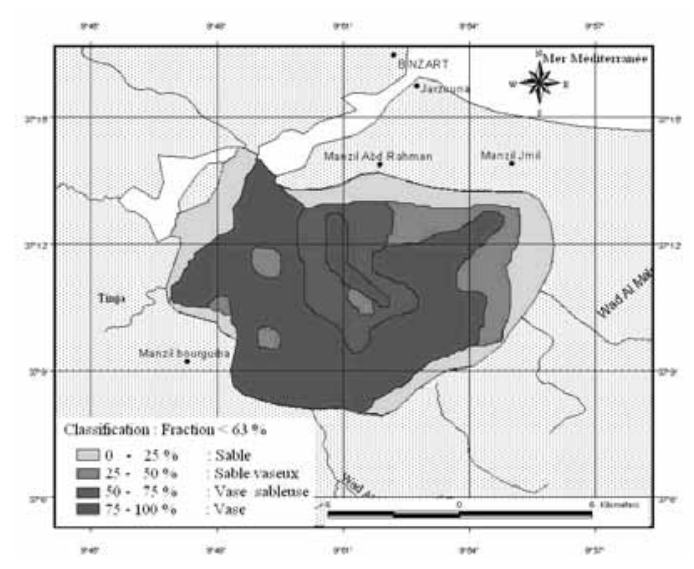

Figure 7. Répartition spatiale des rapports grossiers/fins.

- (2) La zone centrale est caractérisée par de la vase sableuse. C’est un mélange de vase et de sables fins. Cette zone est cernée par de la vase à l'exception du nord où elle passe à des sables fins du côté de Menzel Abderrahmen.

- (3) Le long des rives du lac, les sédiments ont généralement une granulométrie grossière. Les pourcentages de sables y sont les plus élevés et dépassent $75 \%$. Ils présentent une granulométrie moyenne de l’ordre de $250 \mu \mathrm{m}$.

- (4) Un faciès sablo-vaseux occupe une zone à l'est de la lagune avec un pourcentage de sable $(120 \mu \mathrm{m}$ < diamètre moyen $<250 \mu \mathrm{m})$ variant entre 25 et $50 \%$.

Nos résultats concernant les analyses granulométriques sont en relative concordance avec ceux publiés par KAMENS et al. (1984), SOUSSI et al. (1983), ANONYME (2002), ROMDHANE \& BELKHOUJA (2004) et OUAKAD (1993 et 2007). En effet les types de faciès ont été identifiés en fonction de la morphologie des fonds et surtout des sources d'apport. Les faciès argileux occupent toute la zone centrale, ouest et sudouest de la lagune. Ces zones constituent des pièges des sédiments fins issus des apports fluviaux lors des crues et qui se déposent soit par excès de charge soit par décantation. $\mathrm{Au}$ fur et à mesure que l'on se dirige vers les zones littorales nord, est et sud de la lagune, la fraction argileuse tend à diminuer et se mêle progressivement à la fraction grossière donnant lieu à l'établissement d'un faciès sablo-vaseux profond (figure 7). Le faciès sableux dominé par la fraction grossière caractérise toutes les zones littorales de la lagune à l'exception de la partie ouest qui se distingue par la fraction fine terrigène dès les plus faibles profondeurs. Cependant, nos résultats montrent une relative évolution de la répartition des sédiments de surface par rapport aux travaux antérieurs présentés par SOUSSI et al. (1983 et 1985) au nord-est de la lagune pendant ces deux dernières décennies. Nos résultats montrent que les vases envahissent davantage la partie est de la lagune, en particulier au niveau de la station de conchyliculture de Menzel Jemil (Station 19) où le pourcentage de la fraction fine est de l'ordre de $75 \%$. Ce serait dû à l'impact des organismes filtreurs, dont l'action agglomère les particules fines. L'observation à la loupe binoculaire de l'échantillon au niveau de la station 19, 
montre que cette vase est riche en pelotes fécales. D'après ROMDHANE \& BELKHOUJA (2004), SRARFI et al. (2004) et SRARFI (2007) les plus fortes teneurs en matière organique sont observées au centre ainsi que dans les parties nord et nord-est de la lagune, région de Menzel Abderrahmen et Menzel Jmil, où les vases dépassent 16\% (zone marquée par des dépôts vaseux de couleur noire dus aux différents rejets, particulièrement les eaux usées). Ces teneurs dépassent de loin celles trouvées par SOUSSI et al (1983) dont la valeur la plus élevée n'excède pas 15\% ; on s’aperçoit que la lagune a subi au cours de ces deux dernières décennies un enrichissement notable en matière organique.

\subsection{Granulométrie de la fraction grossière ( $\mathrm{D}>0,63 \mu \mathrm{m})$}

Pour chaque échantillon, nous avons établi une courbe granulométrique sur un diagramme semi-logarithmique ayant pour abscisse le diamètre $(\mu \mathrm{m})$ et pour ordonnée le pourcentage cumulé des refus.

Les sédiments prélevés dans la lagune de Bizerte sont caractérisés par des courbes cumulatives ayant plusieurs formes :

- (1) Les courbes ayant la forme d'un S à forte pente, bien redressé et bien régulier malgré l'existence de légères différences dans leurs allures (figure 8) et par un faciès parabolique (figure 9). Ce faciès caractérise la quasi-totalité des sédiments prélevés dans les profondeurs comprises entre 8 et $10 \mathrm{~m}$. Une telle forme des courbes montre qu'on a un stock sédimentaire homogène et des conditions d'énergie adaptées à la charge transportée (DESPRAIRIES, 1974 ; LAFOND, 1953 et 1965 ; RIVIERE, 1952, 1953 et 1977).

- (2) Les sédiments (stations 33, 5, 2, 3, 11, 17 et 44) dont les courbes cumulatives semi-logarithmiques sont assimilables à des droites, présentent un faciès logarithmique (figure 10) dans les profondeurs comprises entre $7 \mathrm{~m}$ et $9 \mathrm{~m}$, où la pente du fond est la plus faible. Ce faciès indique un mode de transport par les courants et un dépôt par excès de charge lorsque la vitesse de ces courants diminue (DESPRAIRIES, 1974 ; LAFOND, 1953 et 1965 ; RIVIERE, 1952 et 1953).

- (3) Des sédiments à faciès hyperbolique (figure 11). Ils se sont déposés par décantation dans un milieu calme où l'hydrodynamisme est trop faible pour les remettre en suspension. Ces sédiments sont formés essentiellement par les matériaux fins $(<63 \mu \mathrm{m})$ dans les plus grandes profondeurs (DESPRAIRIES, 1974 ; LAFOND, 1953 et 1965 ; RIVIERE, 1952, 1953 et 1977).

\subsection{Distribution spatiale des sables et mode de transport}

Les sables grossiers sont présents sur une grande superficie de la lagune. Cependant le sable moyen est observé du côté est de la lagune. A l'est de la lagune, quelques endroits sont tapissés par des sables fins et des sables très fins (figure 12). 


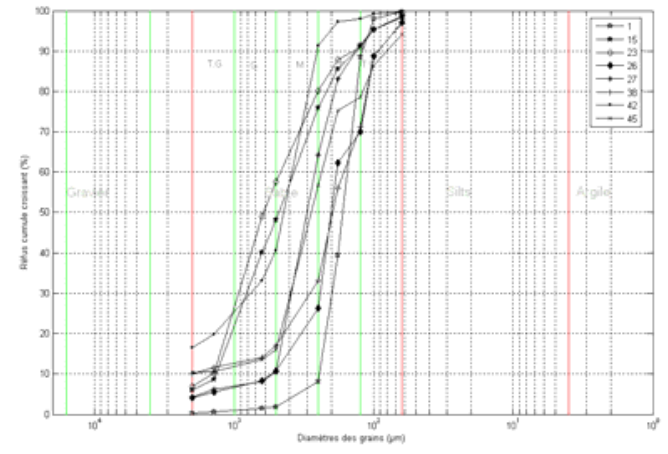

Figure 8. Courbes granulométriques des sédiments superficiels en forme de $S$.

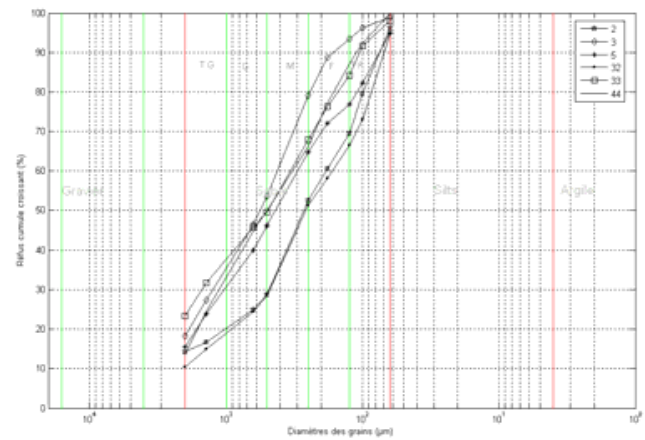

Figure 10. Courbes granulométriques des sédiments superficiels en forme logarithmique.

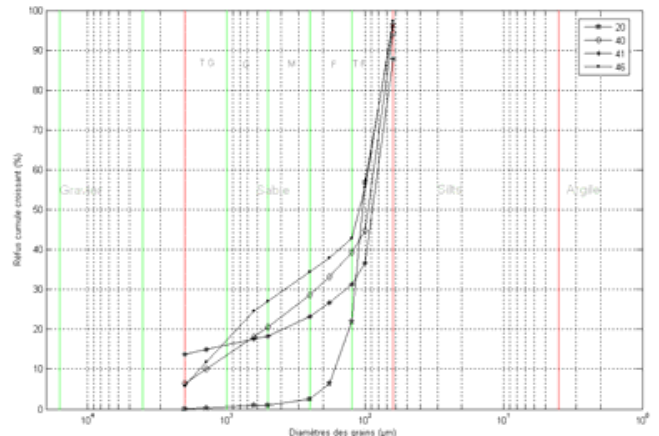

Figure 9. Courbes granulométriques des sédiments superficiels en forme parabolique.

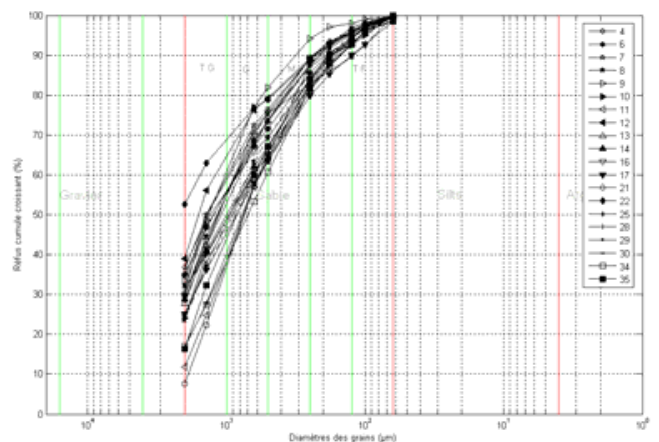

Figure 11. Courbes granulométriques des sédiments superficiels en forme hyperbolique.

\subsection{Mode de transport}

La position des points représentatifs des sédiments superficiels prélevés dans la lagune de Bizerte, sur le diagramme de Passega (PASSEGA, 1957), montre que les valeurs du premier fractile varient entre $149 \mu \mathrm{m}$ et $3391 \mu \mathrm{m}$ et les valeurs de la médiane varient de $95 \mu \mathrm{m}$ à $2100 \mu \mathrm{m}$ (figure 13).

Cette représentation a permis de montrer les différents modes de transport des sédiments dans la lagune de Bizerte :

- un transport des sédiments par saltation au niveau des stations 51 et 1, représenté sur le diagramme de Passega par le segment (RQ),

- un transport par saltation auquel s'ajoutent quelques grains transportés par roulement pour les stations 26, 45, 38 et 40, représenté par le segment (QP) du diagramme de Passega,

- un transport essentiellement par roulement et par suspension gradée représenté par la branche (OP) du diagramme de Passega ; les sédiments correspondant à ce mode de transport sont les sables des stations 42,5 et 44 , 
- un transport exclusivement par roulement (branche ON) caractérisant les sédiments grossiers. Il présente la majorité des autres échantillons.

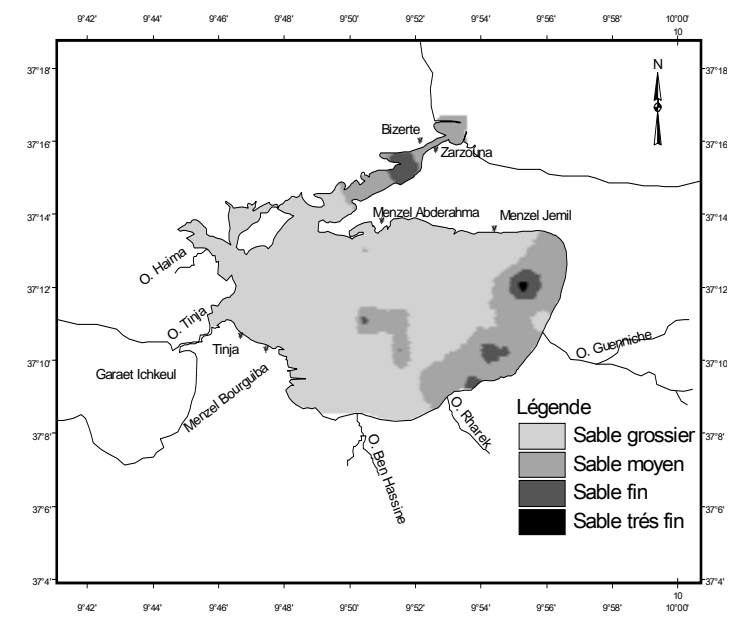

Figure 12. Distribution spatiale des sables.

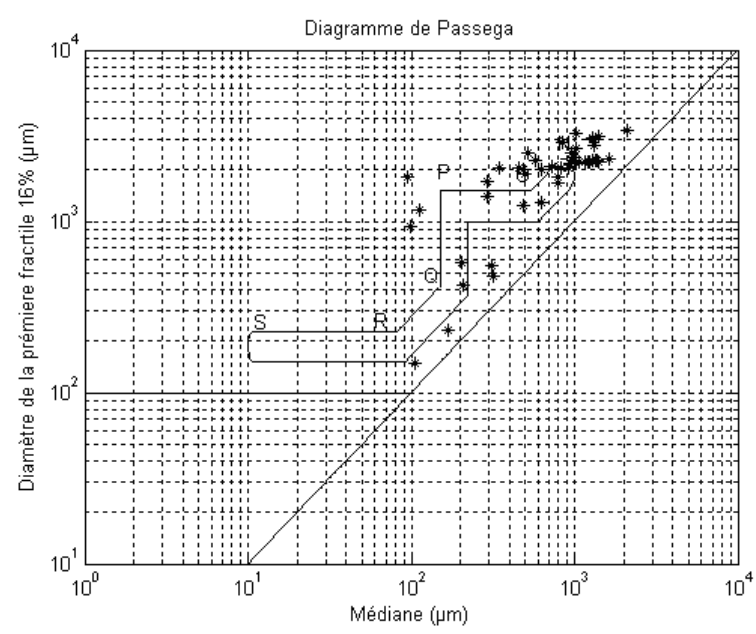

Figure 13. Position des points représentatifs des sables prélevés de la lagune de Bizerte dans le diagramme de Passega.

\subsection{Granulométrie de la fraction fine $(\mathrm{D}<0,63 \mu \mathrm{m})$}

La représentation graphique des courbes cumulatives semi-logarithmiques du diamètre en fonction du pourcentage volumique de la granulométrie de la fraction fine $(<63 \mu \mathrm{m})$ (figure 14) montrent une allure parabolique au niveau de la totalité des échantillons analysés. L'allure parabolique indique que les dépôts sont peu à non évolués et les sédiments sont transportés par un courant de turbidité (BARUSSEAU \& VENECPEYRE, 1973).

A partir du tableau fourni par l'analyse des sédiments fins (diamètre $<63 \mu \mathrm{m}$ ) par le sédimentomètre LASER, nous avons calculé le pourcentage des silts $(>4 \mu \mathrm{m})$ et des argiles $(<4 \mu \mathrm{m})$ dans chaque échantillon. Le pourcentage des argiles dans la totalité des échantillons est compris entre 5 et 20\% (figure 15). La répartition des argiles est localisée dans la zone de déversement de l'Oued Garech au sud et dans la partie centrale de la lagune. Les argiles sont détectées aussi à l'ouest dans la zone de déversement de l'Oued Tinja. Le silt est relativement plus dominant que les argiles. Il est localisé surtout à l'est et au nord-est de la lagune. Il couvre aussi le chenal de navigation jusqu'au Menzel Bourguiba au sud-ouest de la lagune.

Nous constatons que la fraction fine des sédiments est constituée essentiellement par des proportions assez importantes de silts, la fraction argileuse est moins importante. 


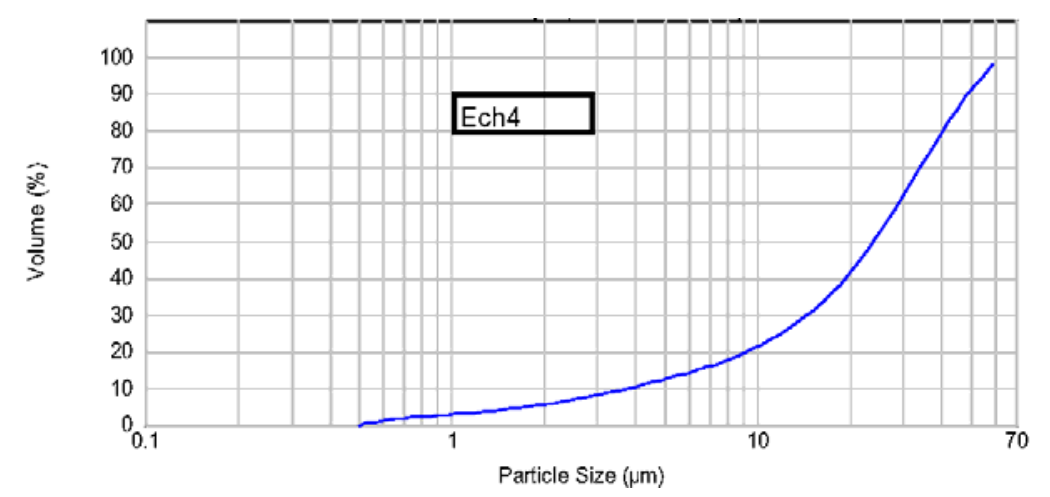

Figure 14. Courbe granulométrique en forme parabolique de la granulométrie fine.

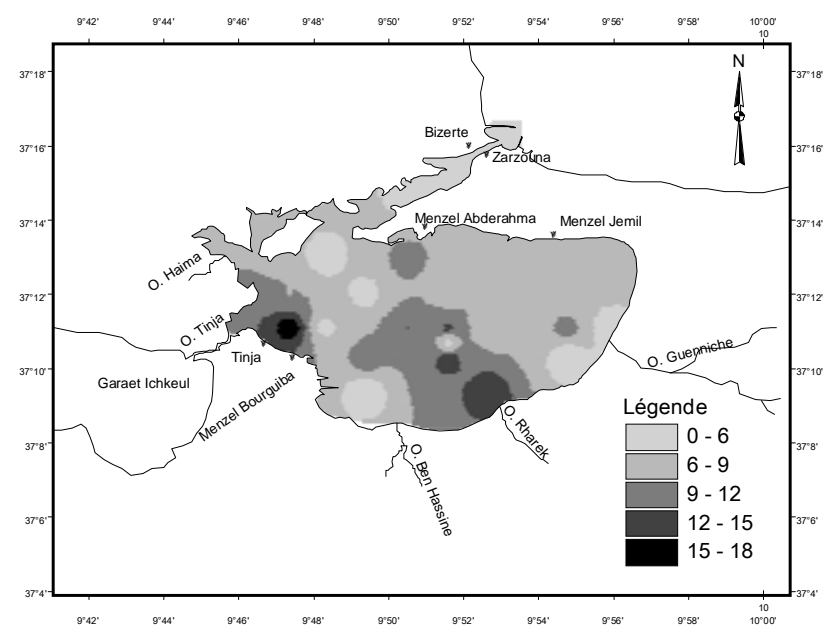

Figure 15. Distribution spatiale des argiles dans la fraction fine.

\section{Hydrodynamique sédimentaire : comparaison avec la modélisation}

Nos résultats d'analyses granulométriques montrent la présence essentielle des sédiments grossiers près des zones côtières en présence des courants de fond assez forts. Au nord de la lagune les zones côtières sont érodées et ceci est dû à l'absence d'apports sableux continentaux et à l'intensité des courants. La formation de l'écran de sable fin observée (figure 12) dans la partie est de la lagune est attribuée à la faible énergie de la houle qui est amortie par les faibles profondeurs au niveau de ce secteur. La faible pente du fond dans la zone sud-est, et la faible tension de cisaillement d'arrachement sont les deux causes essentielles de la présence des sables fins dans cette zone qui ne dépasse pas les 6 mètres de profondeur.

Nos travaux montrent une certaine évolution de la répartition des sédiments de surface de la lagune par rapport aux travaux antérieurs présentés par SOUSSI et al. (1983 et 1985). En effet il est constaté lors de cette étude que les vases couvrent principalement la partie est de la lagune, en particulier au niveau de la station de conchyliculture de Menzel Jemil. Les sédiments de surface prélevés dans cette zone présentent plus de 75\% des sables fins $(<63 \mu \mathrm{m})$. La présence de la fraction fine des sédiments dans cette zone 
est due à l'impact des organismes filtreurs dont l'action agglomère les particules fines. Cette vase est riche en pelotes fécales.

D’après cette étude, nous pouvons conclure que les régions côtières sont des régions à renouvellement intense alors que la région centrale est une région de dépôt.

Un aperçu général sur la taille des grains et le pourcentage du sable fin montre une dominance de la vase, a fortiori la dominance des apports continentaux des bassins versants limitrophes. Les faibles échanges sédimentaires de la lagune avec la mer à travers le goulet sont en faveur d'un envasement de la dépression ellipso-lagunaire de Bizerte.

\section{Conclusion}

L'analyse granulométrique des sédiments de surface dans la lagune de Bizerte révèle une organisation grossièrement concentrique altérée par les apports continentaux. La granulométrie varie, depuis celles des vases situées principalement dans le centre du bassin à celles des sables grossiers périphériques. Nos résultats montrent une certaine évolution de la répartition des sédiments de surface de la lagune par rapport aux travaux présentés par SOUSSI et al. (1983) et SOUSSI et al. (1985). En effet il a été constaté lors de cette étude que les vases couvrent principalement la partie est de la lagune, en particulier au niveau de la station de conchyliculture de Menzel Jemil. L'accumulation des vases dans cette zone est attribuée à la présence d'organismes filtreurs.

La représentation des courbes cumulatives de la granulométrie des sédiments de surface de la lagune montre plusieurs formes d'allure et donc de dynamiques différentes. Les représentations graphiques prennent la forme d'un $S$ peu régulier et étalé et par un faciès parabolique indiquant un stock sédimentaire homogène et des conditions d'énergie adaptées à la charge transportée.

Au niveau des profondeurs moyennes (entre 4 et $8 \mathrm{~m}$ ) et dans la zone est où la pente du fond est la plus faible les sédiments présentent des courbes cumulatives droites qui indiquent un mode de transport par les courants de turbidité et un dépôt par excès de charge lorsque la vitesse de ces courants diminue.

Au centre de la lagune et dans les fortes profondeurs la sédimentation s'effectue par excès de charge quand les courants sont faibles et les courbes cumulatives des échantillons de surface présentent une forme hyperbolique.

Selon la représentation des indices granulométriques des sédiments analysés de la lagune de Bizerte l'origine des sables est essentiellement continentale. Ces sables sont transportés par les oueds du bassin versant de la lagune.

La fraction fine des sédiments est constituée essentiellement par des proportions assez importantes de silts, la fraction argileuse est d'importance moindre.

La dynamique sédimentaire dans la lagune de Bizerte est liée essentiellement aux courants qui sont dus aux vents dominants de secteurs ouest et nord-ouest et par les apports continentaux du bassin versant. 
Étude de l'hydrodynamique sédimentaire de la lagune de Bizerte : 11.13

\section{Références bibliographiques}

ANONYME (2002). Etat actuel et évolution de l'exploitation halieutique et aquacole des lagunes (Bizerte). Rapport final. INSTM. Tunisie. 133p

ANPE -Agence Nationale de Protection de l'Environnement- (1990). Étude préliminaire de l'écologie du lac de Bizerte. Rapport ANPE, Tunis. 100 p.

BARUSSEAU J.P., VENEC-PEYRE M.T. (1973). Interprétation des vasières de la plateforme Sud-Armoricaine (Golfe de Gascogne). C.R. Acad. Sci. Paris, 276-D. 18, pp 2505-2508.

BEJAOUI B. (2009). Développement d'un modèle tridimensionnel couplé dynamiqueécologie : application à la lagune de Bizerte. Rapport de thèse de spécialité en génie hydraulique. ENIT, Département d'hydraulique, $210 \mathrm{p}$.

BEJAOUI B., HARZALLAH A. (2005). Modèle hydrodynamique de la lagune de Bizerte avec forçage saisonnier. Atelier de Modélisation des Écosystèmes Marins, organisé par l'INSTM, Tunis.

BEJAOUI B., HARZALLAH A., MOUSSA M., CHAPELLE A. (2008). Modèle couplé dynamique-écologie pour la lagune de Bizerte. Bull de l'Inst. Nat. des Sci. et Tech. de la Mer de Salammbô, Vol. 35, pp 131-148.

BLUMBERG A.F., MELLOR G.L. (1987). A description of a three-dimensional coastal ocean circulation model, in Three-Dimensional Coastal Ocean Models. Coastal Estuarine Sci., AGU, Vol. 4, Edited by N. S. Heaps, pp 1-16. doi:10.1029/C0004p0001 DESPRAIRIES A. (1974). Degré de représentativité des groupes de grains dans le rythme. Bull. Soc. Geol. France, Vol. 7(16), 624 p.

DGBGTH (2006). Base de données SOUDOUD. Ministère de l'Agriculture.

GIMAZANE J.P. (1981). La production de la moule au lac de Bizerte. Bull. Off. Natn. Pêche, n ${ }^{\circ}$, pp 128-129.

GREDOPAR -Groupe d'étude du domaine paralique- (1986). Projet régional méditerranéen d'aquaculture : évaluation des potentialités aquacoles du lac de Bizerte. FAO/Medrap, Septembre 1986, pp 1-42.

HARZALLAH A. (2003). Transport des polluants dans la lagune de Bizerte simulé par un modèle de circulation de l'eau. Bull de l'Inst. Nat. des Sci. et Tech. de la Mer de Salammbô, Vol. 30, pp 115-133.

HIDROTECNICA PORTUGUESA (1995). Étude générale pour la protection du littoral tunisien contre l'érosion marine, Ministère de l'Équipement et de l'Habitat. KALLEL M.R. (1989). Hydrologie du lac de Bizerte. Rapp. Int. DGRE. Tunis, 41 p. KAMENS J., PILKEY O., WHALTING P. (1984). Étude sédimentologique des lacs de Bizerte et de Tunis. Notes Serv. Géo. Off. Nat. Min., n 49, pp 5-25.

KAMENS J., PILKEY O., HERON D., HARBRIDGE W., KEER F., THORNTON S. (1989). The sedimentology of three Tunisian lagoons. Marine Geology, Vol. 88, pp 285-301. doi:10.1016/0025-3227(89)90102-3 
LAFOND L.R. (1953). Sur l'évolution granulométrique des sédiments dans le bassin de la Vilaine, Bull. Soc. Geol. France, Vol. 6, 75 p.

LAFOND L.R. (1965). Études littorales et estuariennes en zone tropicale humide. Thèse de Doctorat d'État, Orsay.

OUAKAD M. (1982). Évolution sédimentologique et caractères géochimiques des dépôts récents de la Garaât Ichkeul (Tunisie septentrionale). Thèse de Doctorat de Spécialité. Université de Perpignan, 178 p.

OUAKAD M. (1993). Caractères sédimentologiques et géochimiques des dépôts superficiels de la lagune de Bizerte (Tunisie septentrionale) in : circulation des eaux et pollution des côtes méditerranéennes des pays du Maghreb. Edition INOC, Izmir, Turquie, pp 187-194

OUAKAD M. (2007). Genèse et évolution des milieux laguno-lacustres du Nord-Est de la Tunisie (Garaet el Ichkeul, lagune de Bizerte et de Ghar el Melh). Thèse d'état. Univ. el Manar, Tunis. $461 \mathrm{p}$

PASSEGA R. (1957). Texture as characteristic of clastic deposition. Ann. Assoc. Petrol. Géol. Vol. 41, pp 1952-1984.

RIVIERE A. (1952). Expression analytique générale de la granulométrie des sédiments meubles. Bull. Soc. Geol. France, Vol. 61(2), 155 p.

RIVIERE A. (1953). Méthode d'interprétation de la granulométrie des sédiments meubles. Revue de l’Institut français du pétrole, Numéro spécial, pp 102-107.

RIVIERE A. (1977). Méthodes granulométriques technique et interprétation. Edit. Masson, Paris, 167 p.

ROMDHANE M.S., BELKHOUJA H. (2004). Interaction entre la qualité du substrat et la faune malacologique de la lagune de Bizerte. Final report research promotion program, pp 115-124.

SOUSSI N. (1981). Mécanismes de la sédimentation et évolution paléogéographique de la lagune de Bizerte (Tunisie) durant le quaternaire récent. Thèse de Doct. Spéc, Uni. Toulouse, 229 p.

SOUSSI N., LEVY A., ZAOUALI J. (1983). La lagune de Bizerte : Sédimentologie et écologie des Foraminifères et Mollusques. Off. Nat. Mines. Notes Serv. Géol. Tunisie, $\mathrm{n}^{\circ}$ 47, pp 27-40.

SOUSSI N., ALOISI J.C., GOT H., MONACO A. MIRABILE L. (1985). Reconnaissance du remplissage sédimentaire de la lagune de Bizerte par sismique réflexion haute résolution. Notes du Service Géologique de Tunisie, 51 p.

SRARFI F. (2007). Étude géochimique et état de pollution de la lagune de Bizerte. Thèse de doctorat, Université El Manar. Tunis. 122 p.

SRARFI F., SLIM SHIMI N., TAGOURTI M.A. (2004). Les rejets anthropiques et leur influence directe sur l'évolution actuelle de la lagune de Bizerte. Notes du Service Géologique, Tunisie, nº 72, pp 55-64. 